\title{
AiMT
}

Advances in Military Technology

Vol. 15, No. 1, 2020, pp. 149-162

ISSN 1802-2308, eISSN 2533-4123

DOI 10.3849/aimt.01329

\section{The Covariance Matrix Analysis and Statistics for Near-field Sources Localization Model}

\author{
F. Nebus* and S. Gažovová \\ Armed Forces Academy of General M. R. Štefánik, Liptovský Mikuláš, Slovak Republic
}

The manuscript was received on 23 April 2019 and was accepted after revision for publication as research paper on 13 March 2020.

\begin{abstract}
:
The paper presents the mathematical model of the localization of electromagnetic sources in near-field region based on the sources localization mathematical model valid for the far-field region. The aim of the article is to show similarities and differences between both models with a deeper focus on near-field region model analysis using planar equidistant sensors array. Although both concepts in a high-level mathematical description apparatus look very much the same, in details the near-field region model reconstruction process is more complex with different constrains. Detailed covariance matrix analysis and statistics of the covariance matrix represents the main part of the article. In the conclusion, paper shows some model verification results for the localization of single source, correlated sources and coherent sources.
\end{abstract}

\section{Keywords:}

autocorrelation, covariance matrix, cross-correlation, near-field region, source localization

\section{Introduction}

This paper is an extension of work originally presented in New Trends in Signal Processing 2018 [1]. The original article was focused on the sensors signal covariance matrix analysis of near-field region and on similarities and differences between nearand far-field models based on the "harmonics parameters retrieval" and consequences to the signal source parameters estimation. The sensors signals covariance matrix analysis was performed for the uniform linear sensor array.

In this article, we present a general model description based on the planar equidistant sensor array, which gives a more complex view on the used algorithms and overall signal processing in comparison to linear sensor array. The paper presents

\footnotetext{
* Corresponding author: Department of Electronics, Armed Forces Academy of General M. R. Štefánik, Demänová 393, SK 03106 Liptovský Mikuláš, Slovak Republic. Phone: +421960423 884,E-mail: frantisek.nebus@aos.sk
} 
a common problem in the radio-signal processing from the planar sensors array. Appropriate signal component selection according to defined sources parameters with following sensors array signal covariance matrix processing addresses signal sources parameters estimation (i.e. azimuth, elevation, distance, position). In our model, each sensors array contains omnidirectional sensors only, which are not interacting one to each other. General approach for the far- and near-field problem solution is the same, however particular steps are dependent on each simplified mathematical model including constrains, which were used for the selected real scenario. The mathematical descriptive apparatus of the signal sensors covariance matrix (Eq. (2) and Eq. (6)) and the decomposition algorithms (Eq. (19) and Eq. (20)) in near and far-field models look very much the same for the uniform linear sensor array and for the uniform planar sensor array, too. The differences are in the structure of bearing vectors and matrixes forming the sensors signals covariance matrix, which makes the covariance matrix much more complicated and complex in its structure.

The main reason for an accurate localization of electromagnetic interference (EMI) sources is to develop an effective technology for the evaluation and localization of EMI sources. Recently, electromagnetic compatibility (EMC) has played an important role in electronic devices design. One of the options for the EMI sources localization in the near-field region is to apply the methods and algorithms used for EMI sources localization valid for the far-field region.

\section{Model Definition}

\subsection{Far-field Model}

All conventional and high-resolution models of the sources localization in far-field region are derived from the covariance matrix description and processing. They are well suited for digital signal processing with high accuracy and resolution. Practical utilization depends on algorithms of how to retrieve sources angular information (sources localization) from the covariance matrix of intercepted sensors signal compound in classical methods (i.e. Beamforming), criterial and super resolution methods (i.e. MUSIC - Multiple Signal Classification) [2].

The basis for the localization in far-field model is the planar electromagnetic wave structure. The signal received from $D$ harmonic signals sources $s l$ by $m, n$-th sensor in the sensor array, defined with $M$ sensors in x-axis direction and $N$ sensors in $\mathrm{y}$-axis direction, is given

$$
x_{m, n}(i)=\sum_{l=1}^{D} s_{l}(i) \mathrm{e}^{\mathrm{j} \frac{2 \pi d}{\lambda_{l}}\left[m \sin \left(\phi_{l}\right) \sin \left(\theta_{l}\right)+n \sin \left(\phi_{l}\right) \cos \left(\theta_{l}\right)\right]} \Psi_{m, n}^{l}+v_{m, n}(i)
$$

where $i$ is the signal sample index in time, $d$ is the inter element sensor distance of the equidistance planar sensor array, $\lambda_{l}$ is the wavelength of $l$-th source signal, $\varphi_{l}$ is the azimuth angle of direction of arrival of $l$-th source, $\theta_{l}$ is the elevation angle of direction of arrival of $l$-th source, $\Psi_{m, n}^{l}$ is the sensor pattern function value of $m, n$-th sensor for $l$-th source and $v_{m, n}$ represents additive noise for $m, n$-th sensor.

The signals received by all sensors from planar sensor array create the signal matrix with dimension $M \times N$. The signal matrix is transformed according to particular algorithm into the vector $\boldsymbol{x}(i)$ with $M \cdot N \times 1$ dimension. The covariance matrix $\boldsymbol{W}$ created from the signal vector $\boldsymbol{x}(i)$ is defined as given 


$$
\boldsymbol{W}=E\left\{\boldsymbol{x}(i) \boldsymbol{x}(i)^{\mathrm{H}}\right\}=E\left\{(\boldsymbol{A s}(i)+\boldsymbol{v}(i)) \cdot(\boldsymbol{A s}(i)+\boldsymbol{v}(i))^{\mathrm{H}}\right\}=\boldsymbol{A} \boldsymbol{W}_{s} \boldsymbol{A}^{\mathrm{H}}+\boldsymbol{\sigma}^{2} 1
$$

where $s(i)$ is the sources vector, matrix $\boldsymbol{A}$ represents the delays with respect to the source $l$ and $m$ and $n$ position of sensor, $v(i)$ is the additive white noise vector with average power $\sigma^{2}, \boldsymbol{W}_{s}$ is the correlation matrix of the signal sources, 1 is the matrix with ones on main diagonal, symbol ()$^{\mathrm{H}}$ represents Hermitian transpose, $E$ is the mean value operator. Required is matrix $\boldsymbol{A}$, "directional" unique steering matrix created from signal "directional" vectors in column form defined by $M \times N$ sensor array elements positions and signals angles of arrival parameters for the time being unknown and it is given as

$$
A=\left[a_{1}, a_{2}, \ldots, a_{D}\right]
$$

where $\boldsymbol{a}_{l}$ for $l$-th source is defined as

$$
\boldsymbol{a}_{l}=\left[\Psi_{1,1}^{l}, \ldots, \mathrm{e}^{\mathrm{j} \frac{2 \pi d}{\lambda_{l}}\left[(M-1) \sin \left(\phi_{l}\right) \sin \left(\theta_{l}\right)+(N-1) \sin \left(\phi_{l}\right) \cos \left(\theta_{l}\right)\right]} \Psi_{M, N}^{l}\right]^{\mathrm{T}}
$$

The general precondition for this model is that the number of signals sources $D$ is smaller than the number of sensors $(M \cdot N)$ of the planar equidistant sensor array $[2,3]$. The precondition for the planar equidistant sensor array is that $\Psi_{m, n}^{l}=1$ for each $m, n$ and $l$.

\subsection{Near-field Model}

The basis for the localization in near-field model is Hertzian dipole theory. The general condition for this model is that the distance between sources and the planar equidistant sensor array is in radiative near-field region defined in [1]. The $l$-th source is described by its coordinates $\left[x^{l}, y^{l}, z^{l}\right]$ and its dipole moment $\boldsymbol{p}_{0}^{l}$ with components $\left[p_{\mathrm{x}}{ }^{l}, p_{\mathrm{y}}{ }^{l}, p_{\mathrm{z}}{ }^{l}\right]$ and the condition is $z^{l}=0$ and $p_{\mathrm{z}}{ }^{l}=0$. The signal received from $D$ sources by $m, n$-th sensor in the sensor array, defined with $M$ sensors in x-axis direction and $N$ sensors in $y$-axis direction with respect to the far-field model, is magnetic intensity field function defined as

$$
\boldsymbol{H}_{m, n}\left(\boldsymbol{r}_{m, n}, i\right)=\sum_{l=1}^{D} \frac{\mathrm{e}^{-\mathrm{j} k^{l} r_{m, n}^{l}}}{4 \pi r_{m, n}^{l}}\left(\mathrm{j} k^{l}+\frac{1}{r_{m, n}^{l}}\right) \mathrm{e}^{\mathrm{j} \omega^{l} i} \mathrm{j} \omega^{l}\left(\boldsymbol{p}_{0}^{l} \times \frac{\boldsymbol{r}_{m, n}^{l}}{r_{m, n}^{l}}\right) \Theta_{m, n}(l)
$$

where $r_{m, n}^{l}$ represents the distance between $m, n$-th sensor position to the dipole centre of $l$-th source position, $i$ is the signal sample index in the time, $k^{l}$ is the wavenumber of $l$-th source, $\omega^{l}$ is the angular frequency of $l$-th source and $\Theta_{m, n}^{l}$ is the sensor pattern function value of $m, n$-th sensor for $l$-th source $[4,5]$.

Covariance matrix created from signals received by all sensors of the planar equidistant sensor array measured for selected polarization dimension $\{x\}$ and $\{y\}$ is in the symbolic form defined by [4] as

$$
\begin{aligned}
& \boldsymbol{W}_{\{\mathrm{x}, \mathrm{y}\}}=E\left\{\boldsymbol{H}_{\{\mathrm{x}, \mathrm{y}\}} \cdot \boldsymbol{H}_{\{\mathrm{x}, \mathrm{y}\}}^{\mathrm{H}}\right\}=E\left\{\boldsymbol{A}_{\{\mathrm{x}, \mathrm{y}\}} \cdot \boldsymbol{P}_{\{\mathrm{y}, \mathrm{x}\}} \cdot \boldsymbol{P}_{\{\mathrm{y}, \mathrm{x}\}}^{\mathrm{H}} \cdot \boldsymbol{A}_{\{\mathrm{x}, \mathrm{y}\}}^{\mathrm{H}}\right\} \\
& \boldsymbol{W}_{\{\mathrm{x}, \mathrm{y}\}}=E\left\{\boldsymbol{A}_{\{\mathrm{x}, \mathrm{y}\}} \cdot \boldsymbol{C}_{\{\mathrm{y}, \mathrm{x}\}} \cdot \boldsymbol{A}_{\{\mathrm{x}, \mathrm{y}\}}^{\mathrm{H}}\right\}
\end{aligned}
$$


where $\boldsymbol{P}_{\{\mathrm{y}, \mathrm{x}\}}$ is the vector of signals sources dipole moments, $\boldsymbol{C}_{\{\mathrm{y}, \mathrm{x}\}}$ is the covariation matrix of sources signals and $\boldsymbol{A}_{\{\mathrm{x}, \mathrm{y}\}}$ is the unique matrix of $D$ sources signals potential vectors given by $M \times N$ elements sensor array positions and sources positions for the time being unknown and is defined in Eq. (3) and $\boldsymbol{a}_{l}$ for $l$-th source is defined as

$$
\boldsymbol{\alpha}_{l}=\left[\begin{array}{c}
\frac{\mathrm{c} \cdot h}{\lambda^{l}}\left(\mathrm{j} \frac{\mathrm{e}^{-\mathrm{j} k^{l} r_{0,0}^{l}}}{2\left(r_{0,0}^{l}\right)^{3}}-\frac{\pi \mathrm{e}^{-\mathrm{j} k^{l} r_{0,0}^{l}}}{\lambda^{l}\left(r_{0,0}^{l}\right)^{2}}\right) \Theta_{0,0}^{l} \\
\vdots \\
\frac{\mathrm{c} \cdot h}{\lambda^{l}}\left(\frac{\mathrm{e}^{-\mathrm{j} k^{l} r_{M-1, N-1}^{l}}}{2\left(r_{M-1, N-1}^{l}\right)^{3}}-\frac{\pi \mathrm{e}^{-\mathrm{j} k^{l} r_{M-1, N-1}^{l}}}{\lambda^{l}\left(r_{M-1, N-1}^{l}\right)^{2}}\right) \Theta_{M-1, N-1}^{l}
\end{array}\right]
$$

where $\mathrm{c}$ is the speed of light, $h$ is the distance between the measured object and the sensor array, $\lambda^{l}$ is the wavelength of $l$-th source and $r_{m, n}^{l}$ is the distance between $m, n$-th sensor position to the dipole centre of $l$-th source.

The general precondition for this model is that the number of sources signals $D$ is smaller than the number of sensors in the planar equidistant sensor array and $\Theta_{m, n}^{l}=1$ for each $m, n$ and $l$.

\section{Covariance Matrix Analysis}

The mathematical models of covariance matrix $\boldsymbol{W}$ represented with Eq. (2) and Eq. (6) are equal in matrix representation, except the additional noise in Eq. (2). There a couple of methods how to estimate sources localization parameters from the covariation matrix $\boldsymbol{W}$. These methods have different accuracy, resolution and stability since the problem is stationary stochastic in nature [2].

For the next analysis, let us assume a general situation, that the sources signals $s_{1}(i), \ldots, s_{G}(i), \ldots, s_{D}(i), Q>D>G$, where $Q$ is the number of sensors in sensor array with $M$ sensors along x-axis and $N$ sensors along y-axis, $G$ represents coherent or partially correlated sources from whole $D$ sources set with correlation $\boldsymbol{b}=\left[b_{1}, \ldots, b_{G}\right]$

$$
s_{l}(i)=b_{l} s_{1}(i)
$$

where $l=1, \ldots, G$.

In the situation for the near-field model, the sources signals are represented by the elements $p_{\mathrm{x}}$ and $p_{\mathrm{y}}$ of dipole moments $\boldsymbol{p}$ not by $s$, but the conditions remain the same.

\subsection{Far-field Covariance}

Generally, the covariance matrix $\boldsymbol{W}$ in far-field has symmetrical Toeplitz structure for linear sensor array in the situation of non-coherent signals situation. The matrix $\boldsymbol{W}$ has Hermitian structure only in the situations with planar sensor array [6, 7].

The covariance coefficient $w_{u, v}$ in the far-field situation is dependent on the angular values of each signal source and the distance between selected couple of sensors in planar equidistant sensor array for far-field situation. The mean value in the time from the covariance coefficient $w_{u, v}$ sample series is defined as 


$$
\begin{aligned}
w_{u, v}= & P_{1} B_{u} B_{v}^{\mathrm{H}}+ \\
& +E\left\{s_{1}(i) B_{u}\left(\sum_{l=G+1}^{D} s_{l}(i) \mathrm{e}^{-\mathrm{j} d_{v} \alpha_{l}}\right)^{\mathrm{H}}\right\}+\mathrm{E}\left\{s_{1}(i) B_{v}^{H}\left(\sum_{d=G+1}^{D} s_{l}(i) \mathrm{e}^{-\mathrm{j} d_{u} \alpha_{l}}\right)\right\}+ \\
& +E\left\{\left(\sum_{l=G+1}^{D} s_{l}(i) \mathrm{e}^{-\mathrm{j} d_{u} \alpha_{l}}\right)\left(\sum_{l=G+1}^{D} s_{l}(i) \mathrm{e}^{-\mathrm{j} d_{v} \alpha_{l}}\right)^{\mathrm{H}}\right\}+\sigma^{2} \delta_{u, v}
\end{aligned}
$$

where $d$ is the distance between sensors in the planar equidistant sensor array, $\alpha$ is the variable for substitution of sources signals angular parameters (azimuth and elevation) defined in Eq. (1), $B_{u}$ and $B_{v}$ represent the final spatial phase coefficient of coherent signals on selected sensor, $\delta_{u, v}=1$ for $u=v$, otherwise $\delta_{u, v}=0$.

It is important to note that the covariance matrix $\boldsymbol{W}$ is the square matrix $M \cdot N \times$ $M \cdot N$, with respect to the particular planar to linear transformation algorithm of the sensors signal matrix array. When using the planar equidistant sensor array in noncoherent situation, only the last row of Eq. (9) is non-vanishing and convergent. The main diagonal of matrix $\boldsymbol{W}$ consists of the equal real value of sensors power and all known algorithms for matrix $\boldsymbol{W}_{s}$ decomposition and signal sources estimation can be applied there. Due to $M \times N \rightarrow M \cdot N \times 1$ signal matrix transformation, matrix $\boldsymbol{W}$ loses its Toeplitz structure and remains only Hermitian. The spatial smoothing [8] before final reconstruction process is needed in coherent situation.

The phasor distribution representation for planar sensor array is rather difficult. However, an example for the 7 element linear antenna array and one signal source is suitable in Fig. 1. It shows the correlation coefficient phasor $w_{1, n}$ distribution (first row of the correlation matrix $\boldsymbol{W}$ ). The amplitude is constant and phase represents linear angular function as long as array is linear-equidistant.

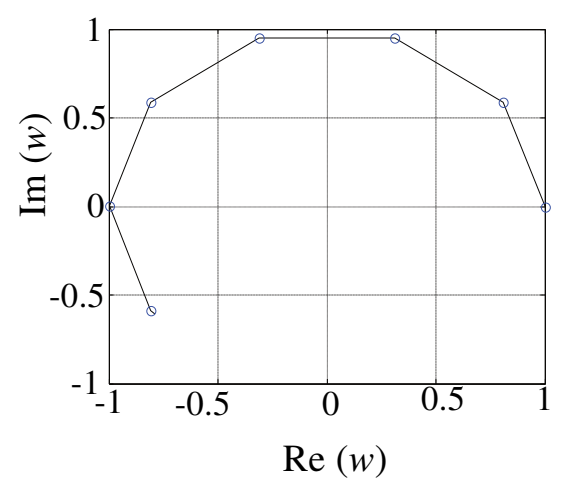

Fig. 1 Normalized correlation coefficient phasor $w_{1, n}$ distribution for the 7 element linear antenna array and one signal source in far-field model

\subsection{Near-field Covariance}

The covariance coefficient $w_{u, v}$ in the near-field situation is dependent on the distance between the position of each source defined by elementary dipole and the position of selected couple of sensors in planar equidistant sensor array. The dependence of distance is shown in Eq. (7). 
The signal from one source acquired on the sensor with $m, n$-th position in the planar equidistant sensor array in polarisation dimension $\{\mathrm{x}\}$ is defined as

$$
h_{\mathrm{x} m, n}(i)=\frac{\mathrm{e}^{-\mathrm{j} k r_{m, n}}}{4 \pi\left(r_{m, n}\right)^{2}}\left(\mathrm{j} k+\frac{1}{r_{m, n}}\right) \mathrm{e}^{\mathrm{j} \omega i} \mathrm{j} \omega h p_{y}
$$

with the conditions $p_{z}=0$ and $z=0$ are true and the distance $r_{m, n}$ is defined as

$$
r_{m, n}=\sqrt{(m d-x)^{2}+(n d-y)^{2}+h^{2}}
$$

where $m$ is the sensor position index along x-axis, $n$ is the sensor position index along $y$-axis, $h$ is the sensor to source plane distance, $x, y$ and $z$ are the coordinates of the source.

The signal compound on the sensor with $m, n$-th position in the planar equidistant sensor array, taking into account only signals generated by the sources in the source plane with respect to the radiative near-field condition, is defined as

$$
\begin{aligned}
h_{m, n}(i) & =\boldsymbol{p}^{1}(i) \sum_{g=1}^{G}\left[b^{g}\left(\mathrm{j} \frac{\mathrm{e}^{-\mathrm{j}\left(k^{g} r_{m, n}^{g}-\omega^{g} i\right)}}{2\left(r_{m, n}^{g}\right)^{3}}\right)-\left(\pi \frac{\mathrm{e}^{-\mathrm{j}\left(k^{g} r_{m, n}^{g}-\omega^{g} i\right)}}{\lambda^{g}\left(r_{m, n}^{g}\right)^{2}}\right)\right]+ \\
& +\sum_{l=G+1}^{D}\left[\boldsymbol{p}^{l}(i)\left(\mathrm{j} \frac{\mathrm{e}^{-\mathrm{j}\left(k^{l} r_{m, n}^{l}-\omega^{l} i\right)}}{2\left(r_{m, n}^{l}\right)^{3}}\right)-\left(\pi \frac{\mathrm{e}^{-\mathrm{j}\left(k^{l} r_{m, n}^{l}-\omega^{l} i\right)}}{\lambda^{l}\left(r_{m, n}^{l}\right)^{2}}\right)\right] \\
h_{m, n}(i) & =\boldsymbol{p}^{1}(i) B_{m, n} \sum_{l=G+1}^{D}\left[\boldsymbol{p}^{l}(i)\left(\mathrm{j} \frac{\mathrm{e}^{-\mathrm{j}\left(k^{l} r_{m, n}^{l}-\omega^{l} i\right)}}{2\left(r_{m, n}^{l}\right)^{3}}\right)-\left(\pi \frac{\mathrm{e}^{-\mathrm{j}\left(k^{l} r_{m, n}^{l}-\omega^{l} i\right)}}{\lambda^{l}\left(r_{m, n}^{l}\right)^{2}}\right)\right]
\end{aligned}
$$

where $B_{m, n}$ represents final correlation given by correlations $b$ and selected $m, n$ sensor spatial position and $\boldsymbol{p}^{l}(i)$ is defined as

$$
\boldsymbol{p}^{l}(i)=\frac{h \boldsymbol{p}_{0}^{l} \mathrm{c}}{\lambda^{l}}
$$

From the $i$-th signal sample at each sensor element of the whole sensor array, one may create matrix $\boldsymbol{H}(i)$ sample with the dimension $M \times N$. The rectangular matrix shall be transformed into the column matrix $M \cdot N \times 1$, but it is important to note that the transformation algorithm has to be applied (from Eq. (6)) accordingly to the "localization" matrix $\boldsymbol{A}$ in Eq. (7).

The mean value from sample series of covariance coefficient $w_{u, v}$ is defined as

$$
\begin{aligned}
w_{u, v}= & h_{u} h_{v}^{\mathrm{H}} \\
w_{u, v}= & {\left[\boldsymbol{p}^{1}(i) B_{u}+E\left\{\sum_{l=G+1}^{D}\left(\boldsymbol{p}^{l}(i)\left(\mathrm{j} \frac{\mathrm{e}^{-\mathrm{j}\left(k^{l} r_{u}^{l}-\omega^{l} i\right)}}{2\left(r_{u}^{l}\right)^{3}}\right)-\left(\pi \frac{\mathrm{e}^{-\mathrm{j}\left(k^{l} r_{u}^{l}-\omega^{l} i\right)}}{\lambda^{l}\left(r_{u}^{l}\right)^{2}}\right)\right)\right\} .\right.} \\
& \cdot\left[\boldsymbol{p}^{1}(i) B_{v}+E\left\{\sum_{l=G+1}^{D}\left(\boldsymbol{p}^{l}(i)\left(\mathrm{j} \frac{\mathrm{e}^{-\mathrm{j}\left(k^{l} r_{v}^{l}-\omega^{l} i\right)}}{2\left(r_{v}^{l}\right)^{3}}\right)-\left(\pi \frac{\mathrm{e}^{-\mathrm{j}\left(k^{l} r_{v}^{l}-\omega^{l} i\right)}}{\lambda^{l}\left(r_{v}^{l}\right)^{2}}\right)\right)\right\}\right]^{\mathrm{H}} .
\end{aligned}
$$


where $r_{u}, r_{v}$ represents the distance between the sensor positon and the dipole centre of source and $B_{u}$ and $B_{v}$ represent the correlation given by correlations in $b$ and selected $u, v$ sensors spatial position.

Eq. (14) can be rewritten into a simplified and more readable form

$$
\begin{aligned}
& w_{u, v}=\left[\boldsymbol{p}^{1}(i)\right]^{2} B_{u} B_{v}^{\mathrm{H}}+ \\
& +E\left\{\boldsymbol{p}^{1}(i) B_{u}\left[\sum_{l=G+1}^{D}\left(\boldsymbol{p}^{l}(i)\left(\mathrm{j} \frac{\mathrm{e}^{-\mathrm{j}\left(k^{l} r_{v}^{l}-\omega^{l} i\right)}}{2\left(r_{v}^{l}\right)^{3}}\right)-\left(\pi \frac{\mathrm{e}^{-\mathrm{j}\left(k^{l} r_{v}^{l}-\omega^{l} i\right)}}{\lambda^{l}\left(r_{v}^{l}\right)^{2}}\right)\right]^{\mathrm{H}}\right\}+\right. \\
& +E\left\{\boldsymbol{p}^{1}(i) B_{v}^{\mathrm{H}} \sum_{l=G+1}^{D}\left[\boldsymbol{p}^{l}(i)\left(\mathrm{j} \frac{\mathrm{e}^{-\mathrm{j}\left(k^{l} r_{u}^{l}-\omega^{l} i\right)}}{2\left(r_{u}^{l}\right)^{3}}\right)-\left(\pi \frac{\mathrm{e}^{-\mathrm{j}\left(k^{l} r_{u}^{l}-\omega^{l} i\right)}}{\lambda^{l}\left(r_{u}^{l}\right)^{2}}\right)\right]\right\}+ \\
& +E\left\{\begin{aligned}
\sum_{l=G+1}^{D}\left[\boldsymbol{p}^{l}(i)\left(\mathrm{j} \frac{\mathrm{e}^{-\mathrm{j}\left(k^{l} r_{u}^{l}-\omega^{l} i\right)}}{2\left(r_{u}^{l}\right)^{3}}\right)-\left(\pi \frac{\mathrm{e}^{-\mathrm{j}\left(k^{l} r_{u}^{l}-\omega^{l} i\right)}}{\lambda^{l}\left(r_{u}^{l}\right)^{2}}\right)\right] \cdot \ldots \\
\quad \ldots\left[\sum_{l=G+1}^{D}\left(\boldsymbol{p}^{l}(i)\left(\mathrm{j} \frac{\mathrm{e}^{-\mathrm{j}\left(k^{l} r_{v}^{l}-\omega^{l} i\right)}}{2\left(r_{v}^{l}\right)^{3}}\right)-\left(\pi \frac{\mathrm{e}^{-\mathrm{j}\left(k^{l} r_{v}^{l}-\omega^{l} i\right)}}{\lambda^{l}\left(r_{v}^{l}\right)^{2}}\right)\right)\right]^{\mathrm{H}}
\end{aligned}\right\}
\end{aligned}
$$

Eq. (15) consists of the same three analytical parts as Eq. (9): coherent - distributed source (upper row), correlated sources (middle part) and non-coherent sources (bottom part).

The representation of the covariance phasor distribution in the near-field is suitable for linear array only, similarly to far-field case. Fig. 2 shows the simplest situation for the correlation coefficient phasor $w_{l, n}$ distribution (first row of the correlation matrix $\boldsymbol{W}$ ) for the 7 element linear antenna array and one signal source. One may see nonlinear covariance coefficients functionality from the linear spacing of the sensors.

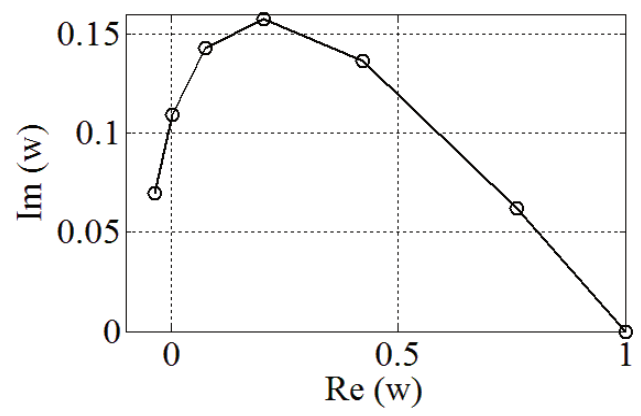

Fig. 2 Near-field model correlation coefficient $w_{1, n}$ distribution for the 7 element linear antenna array and one signal source with coordinates $[0,0,0]$

In the coherent - distributed sources situation, the structure of $\boldsymbol{W}$ is rather more complicated. It is suitable to analyse the last part of Eq. (6), since the matrix $C$ drives 
the final $\boldsymbol{W}$ structure. It consists of the power of sources on the main diagonal and cross covariances between the sources. The matrix $\boldsymbol{C}$ can be written as

$$
\boldsymbol{C}_{\{\mathrm{y}, \mathrm{x}\}}=\boldsymbol{P}_{\{\mathrm{y}, \mathrm{x}\}} \cdot \boldsymbol{P}_{\{\mathrm{y}, \mathrm{x}\}}^{\mathrm{H}}
$$

In case of two coherent sources with the dipole moment, components $\boldsymbol{p}^{1}=\left[p^{1}\right.$, $\left.p^{1}, p^{1}{ }_{\mathrm{z}}\right]$ and $\boldsymbol{p}^{2}=\left[p^{2} \mathrm{x}, p^{2} \mathrm{y}, p_{\mathrm{z}}^{2}\right]$ and $p_{\mathrm{z}}^{1}$ and $p_{\mathrm{z}}^{2}$ are equal to 0 and the cross correlation between $\{x\},\{y\}$ dipole components is equal to 0 . Then the matrix $\boldsymbol{C}$ in polarization dimension $\{x\}$ can be written as

$$
\boldsymbol{C}_{\{\mathrm{x}\}}=\boldsymbol{P}_{\{\mathrm{x}\}} \cdot \boldsymbol{P}_{\{\mathrm{x}\}}^{\mathrm{H}}=\left(\begin{array}{cc}
\left(p_{\mathrm{x}}^{1}\right)^{2} & \left(p_{\mathrm{x}}^{1}\right)\left(p_{\mathrm{x}}^{2}\right)^{\mathrm{H}} \\
\left(p_{\mathrm{x}}^{2}\right)\left(p_{\mathrm{x}}^{1}\right)^{\mathrm{H}} & \left(p_{\mathrm{x}}^{2}\right)^{2}
\end{array}\right)
$$

All three parts of the Eq. (15) are valid and lead into the situation, where the matrix $\boldsymbol{W}$ is non-linear with dumped periodic structure.

From the comparison of far-field and near-field model we can see that in far-field model situation the matrix $\boldsymbol{W}$ has the periodical structure because of the sources signals angular parameters (azimuth and elevation) functionality. The matrix $\boldsymbol{W}$ in the near-field situation is not periodic and it has non-linear properties because the components are not dependent on angular parameters but on the spacing of the sensors and sources. Additionally, in the near-field situation the matrix $\boldsymbol{W}$ does not have Toeplitz structure neither for linear, nor for planar sensor array, it is Hermitian structure only.

The decomposition of $\boldsymbol{W}$ into $\boldsymbol{A}$ and $\boldsymbol{C}$ in non-coherent situation for the far and near-field situation can be performed via known algorithms (i.e. maximum beamforming or MUSIC) [1,2]. Coherent situation in far-field model can be solved with the same algorithms after "spatial smoothing" pre-processing, under specific periodical $W$ structure condition $[6,8]$. Unfortunately, in near-field model such "spatial smoothing" pre-processing is not applicable due to $\boldsymbol{W}$ non-linear properties.

\section{Covariance Matrix Statistics}

In the mathematical model for near-field situation there are some options on how to create the covariance matrix $\boldsymbol{W}$. The first option is to measure the sensors signals for the particular frequency $f$ and then create the covariance matrix $W$ in the time $T$ limited process. The second option is to measure magnetic intensity signals defined in Eq. (5) in the time $T$ limited process, then apply the Discrete Fourier Transform and for particular frequency create covariance matrix $\boldsymbol{W}$. Both options represent real measurement process.

In general form the covariance matrix $\boldsymbol{W}$ element is defined [4] as

$$
W(u, v, f)=\lim _{T \rightarrow \infty} \frac{1}{T} E\left\{H_{T}(u, f) \cdot H_{T}^{\mathrm{H}}(v, f)\right\}
$$

where for the first option $u, v$ are sensors indexes, $H_{T}(u, f)$ is the measured signal for the particular frequency from $u$-th sensor and $H_{T}(v, f)$ is the measured signal for the particular frequency from $v$-th sensor. For the second option $u, v$ are sensors indexes, $H_{T}(u, f)$ is the Fourier transform of $T$ windowed signal $h_{T}(u, t)$ and $H_{T}(v, f)$ is the Fourier transform of $T$ windowed signal $h_{T}(v, t)$.

There are some parameters that can affect the information contained in the covariance matrix $\boldsymbol{W}$. In the stationary stochastic situation, it is the total number of samples 
(in time $T$ ) given by the number of the windows and number of the samples $I$ in each window. The number of samples $I$ is closely related to the sampling frequency $f_{\mathrm{vz}}$. After the signals measurement further DFT processing and the spectral characteristics estimation, the frequency resolution and accuracy are affected by the number of samples and the sampling frequency. It is defined as the ratio of the sampling frequency $f_{\mathrm{vz}}$ to the number of samples $I$ and improves with increasing $I$ or decreasing $f_{v z}$. The information contained in covariance matrix $\boldsymbol{W}$ for selected frequency component is thus dependent from the frequency detection accuracy given by DFT and consequently affects the accuracy of the source localization algorithm. For all poles algorithms i.e. MUSIC small deviations might be critical.

Following examples show four situations how the number of samples and the number of sampling windows affect the covariance matrix $\boldsymbol{W}$ and singular values estimations. All experiments were performed with the number of samples $I=1024$ and frequency accuracy $\pm 12 \mathrm{MHz}$.

In the single non-coherent source situation, the source is defined at the coordinates $[0,0,0] \mathrm{cm}, f=2.45 \mathrm{GHz}$, sensor array as $5 \times 5$ planar equidistant sensor array with $d=2.5 \mathrm{~cm}$, sensor array to source planes distance $h=5 \mathrm{~cm}$. The effect of the number of windows with the same sampling rate on the first five main diagonal elements of the matrix $\boldsymbol{W}$ is shown in Fig. 3a) and on the first three singular value of matrix $\boldsymbol{W}$ is shown in Fig. 3b). The statistics show results for 1 to 50 samples of window. Results show that the main diagonal elements of the matrix $\boldsymbol{W}$ are not affected by the number of window samples and that the singular values converge quickly into fixed values. Statistics shows, that it is only additive noise that influences noise singular values and noise subspace of the matrix $\boldsymbol{W}$. For correct signal detection single sampling window with 1024 samples is good enough.

In the correlated sources situation, we selected two cases with wide and narrow band modulation models. Two sources are at the coordinates $[3,3,0] \mathrm{cm}$ and $[5,2,0] \mathrm{cm}$. Signals are frequency modulated at the carrier frequency $2.45 \mathrm{GHz}$ with different modulation parameters. The power spectrum magnitudes of two sources with frequency modulation are shown in Fig. 4.
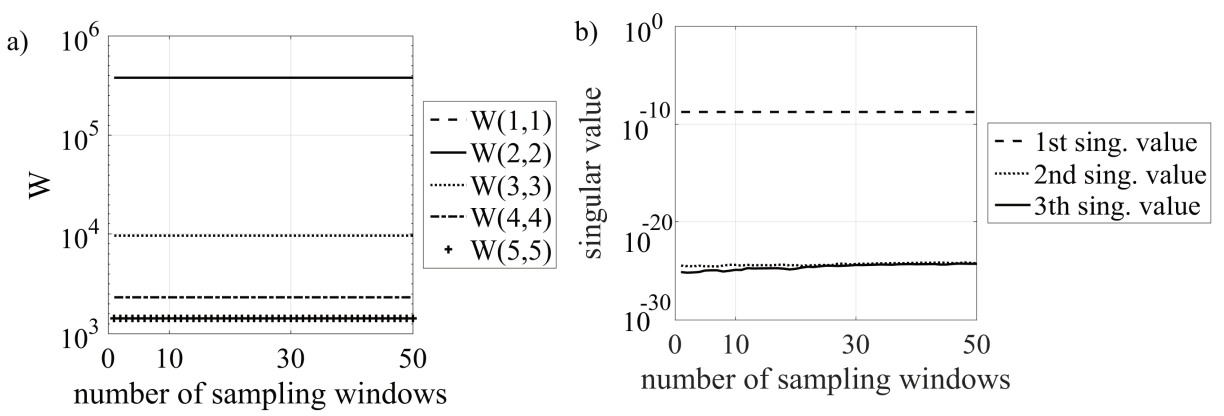

Fig. 3 The statistics results for $x$ polarization of $5 \times 5$ planar equidistant sensor array, a) the first five main diagonal elements of $\boldsymbol{W}$ and $b$ ) the first three singular values of $\boldsymbol{W}$

The first model is with two narrow band frequency modulation signal sources. The effects of the number of windows with the same sampling rate on the information contained in the matrix $\boldsymbol{W}$ for two sources with narrow band frequency modulation are shown in Fig. 5. The effect of the number of windows with the same sampling rate on 
the first five main diagonal elements of the matrix $\boldsymbol{W}$ is shown in Fig. 5a) and on the first three singular values of the matrix $W$ is shown in Fig. 5b). The statistics show results from 1 to 50 samples windows each of 1024 samples of signals. The results show that the singular values converge into the fixed values; the first two singular values signify two sources on detected frequency and the main diagonal elements are not affected by the number of samples windows. The singular values statistics results show that greater number of samples is suitable for the exact sources localization in the narrow band correlated sources situation.
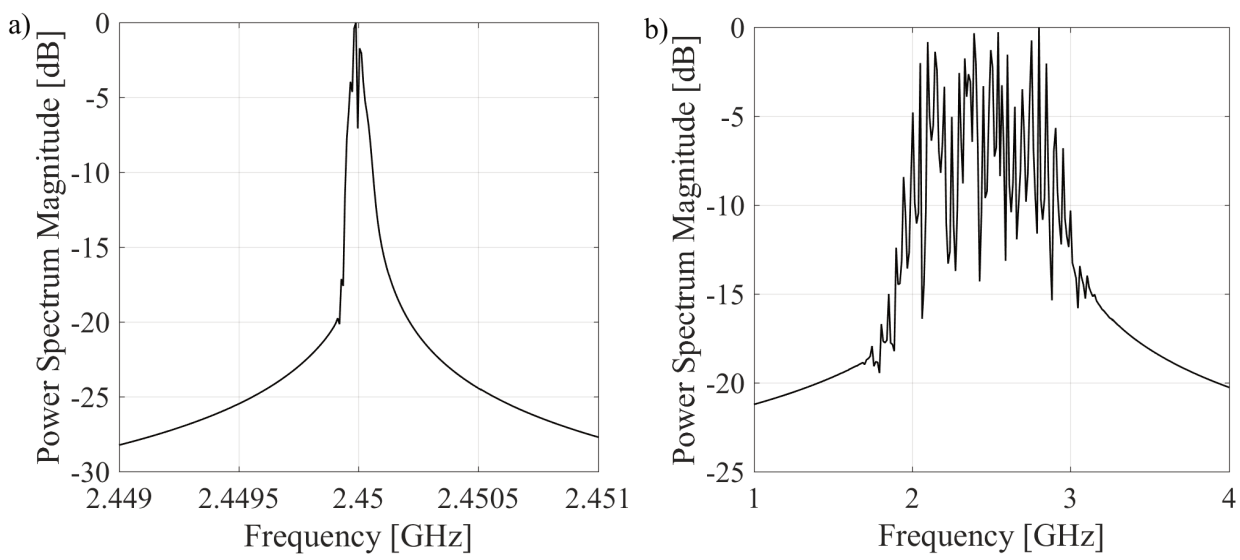

Fig. 4 The power spectrum magnitude of two sources at the coordinates $[3,3,0] \mathrm{cm}$ and $[5,2,0] \mathrm{cm}$ with a) narrow band frequency modulation and $b$ ) wide band frequency modulation NFFT $=1024$
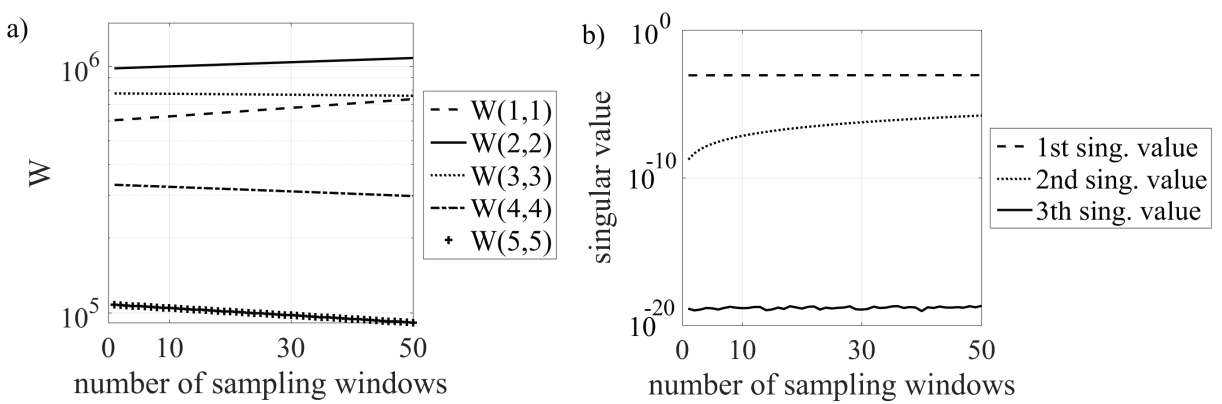

Fig. 5 The statistics results for $x$ polarization of $5 \times 5$ planar equidistant sensor array, for two sources with narrow band frequency modulation a) the first five main diagonal elements of $\boldsymbol{W}$ and $b$ ) the first three singular values of $\boldsymbol{W}$

The second model is with two sources with wide band frequency modulation. The effect of the number of windows with the same sampling rate on the first five main diagonal elements of the matrix $\boldsymbol{W}$ is shown in Fig. 6a) and on the first three singular values of the matrix $\boldsymbol{W}$ is shown in Fig. 6b). The singular values converge into fixed values and the main diagonal elements are not affected by the number of samples windows. The first two singular values signify two sources on detected frequency. For the 
exact sources localization, the suitable number of samples can be lower than in the narrow-band frequency modulation situation.

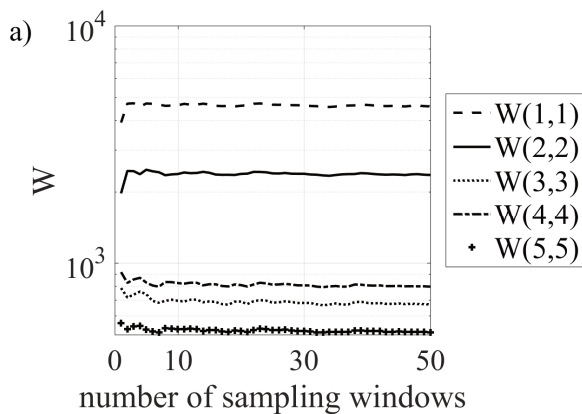

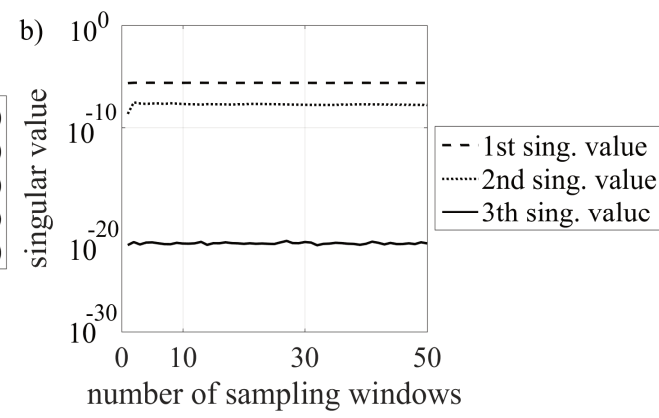

Fig. 6 The statistics results for $x$ polarization of $5 \times 5$ planar equidistant sensor array, for two sources with wide band frequency modulation a) the first five main diagonal elements of $\boldsymbol{W}$ and $b$ ) the first three singular values of $\boldsymbol{W}$

For the coherent sources model two sources are selected, the first one at the coordinates $[2,2,0] \mathrm{cm}$ and the second one at the coordinates $[8,8,0] \mathrm{cm}, f=2.45 \mathrm{GHz}$. The effects of the number of window samples on the information contained in the matrix $\boldsymbol{W}$ for two coherent sources are shown in Fig. 7. The first five diagonal elements of the matrix $\boldsymbol{W}$ are shown in Fig. 7a) and the first three singular values of the matrix $\boldsymbol{W}$ are shown in Fig. 7b).
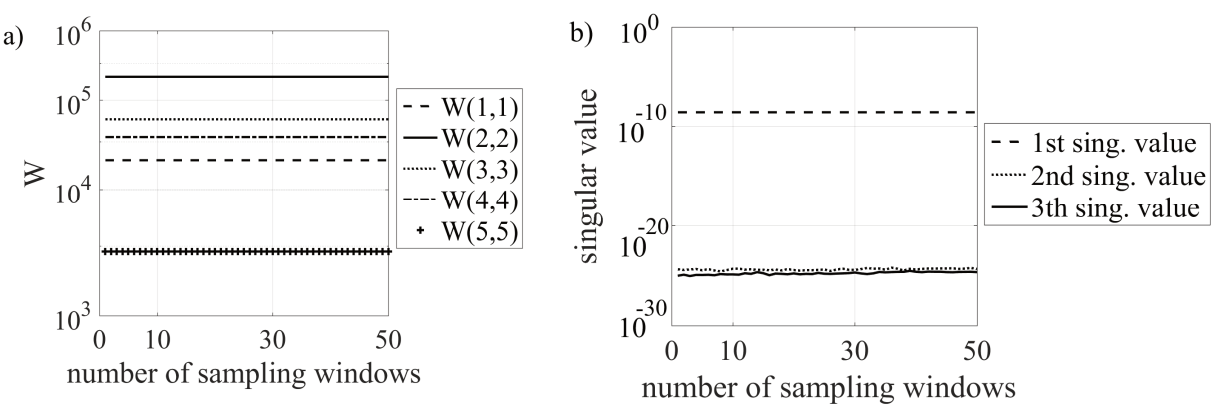

Fig. 7 The statistics results for $x$ polarization of $5 \times 5$ planar equidistant sensor array, for two coherent sources a) the first five main diagonal elements of $\boldsymbol{W}$ and $b$ ) the first three singular values of $\boldsymbol{W}$

Matrix $\boldsymbol{W}$ diagonal elements are not affected by the number of window samples and the singular values characteristics are similar to the single source case. Unfortunately, the singular value distribution for the particular frequency matrix $\boldsymbol{W}$ (indicating the number of sources) indicates only single source situation, since sources are coherent.

\section{Conclusions}

Two localization estimation techniques were selected for the decomposition of the covariance matrix $\mathrm{W}$ for the near-field verification model. The basic one is Beamforming based on formula 


$$
\boldsymbol{P}_{\mathrm{j}}(x, y)=\boldsymbol{A}_{\mathrm{lok}}^{\mathrm{H}} \boldsymbol{W} \boldsymbol{A}_{\mathrm{lok}}
$$

and the second one is MUSIC with search formula

$$
\boldsymbol{P}_{\mathrm{MU}}(x, y)=\frac{1}{\boldsymbol{A}_{\mathrm{lok}}^{\mathrm{H}} \boldsymbol{v}^{\mathrm{H}} \boldsymbol{v} \boldsymbol{A}_{\mathrm{lok}}} .
$$

Classical Beamforming worked as expected with low accuracy and resolution, however was always stable. The MUSIC, based on the eigenvalue decomposition of the matrix with Toeplitz structure representing linear systems, worked for singular value decomposition of non-correlated sources and partially correlated sources well in non-linear near-field model too.

The simulation results for the localization of the single source situation from Fig. 3 are shown in Fig. 8.
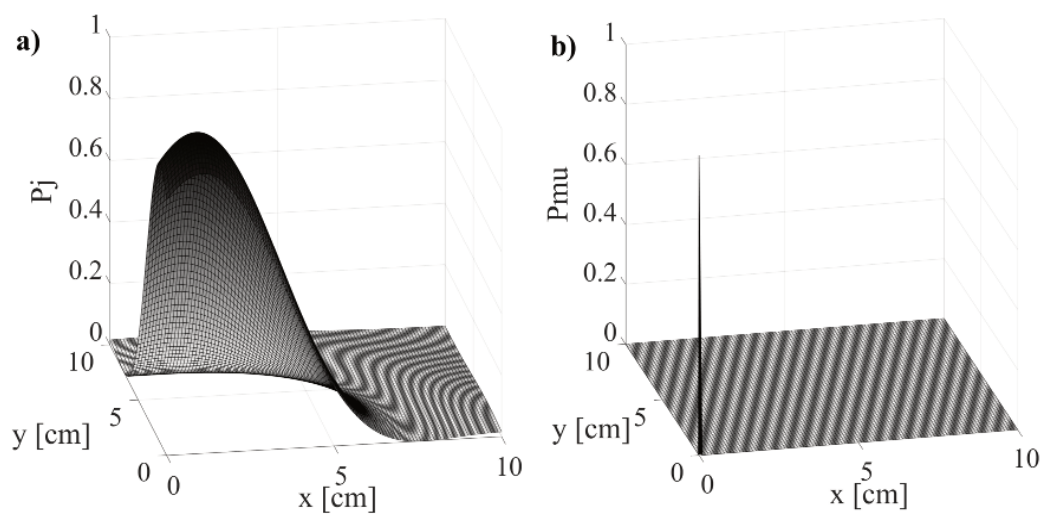

Fig. 8 The simulation results for one single non-coherent source with the centre frequency $2.4492 \mathrm{GHz}$ with the estimation technique a) Beamforming, b) MUSIC

Simulation results for localization of the correlated sources situation with two narrow band frequency modulation sources from Fig. 5 are shown in Fig. 9. All results (narrow and wide-band) showed very good localization results agreement with given sources parameters.
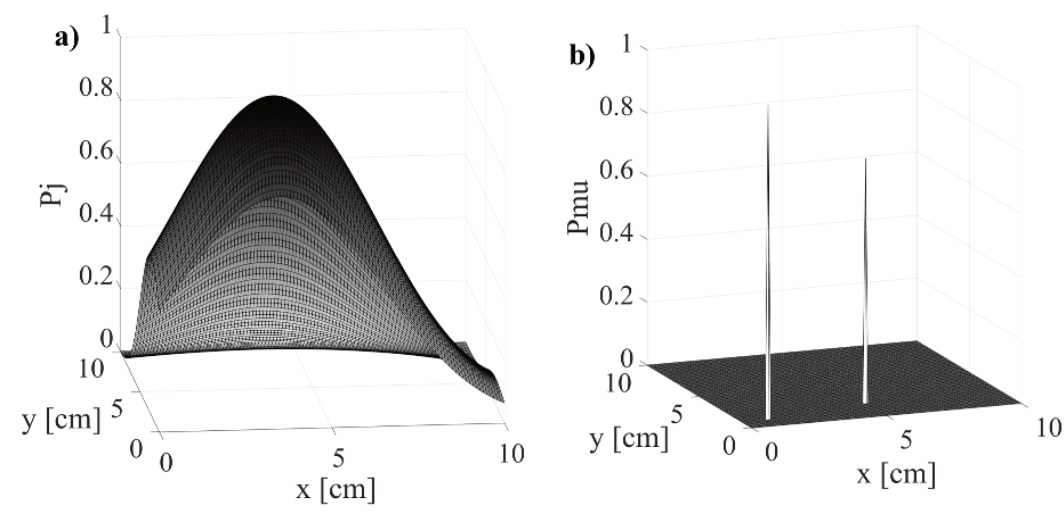

Fig. 9 The simulation results for two correlated sources with narrow-band frequency modulation with the centre frequency $2.4492 \mathrm{GHz}$ with the estimation technique a) Beamforming, b) MUSIC 
Simulation results for the localization of the situation with two coherent sources from Fig. 7 are shown in Fig. 10. Figure shows only one signal source and wrong localization estimation parameters from the covariance matrix $\boldsymbol{W}$ at the frequency component $2.4492 \mathrm{GHz}$.
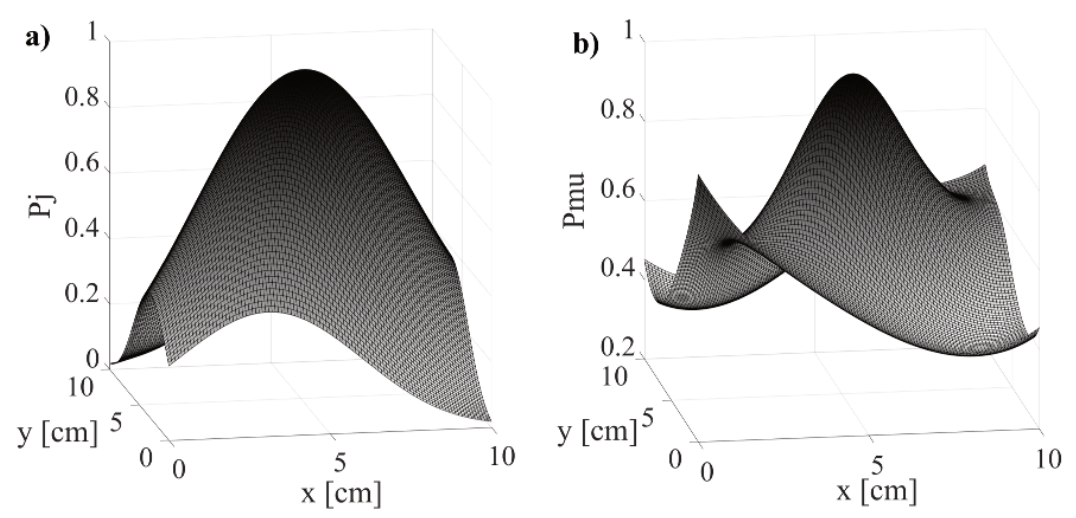

Fig. 10 The simulation results for two coherent sources for frequency $2.4492 \mathrm{GHz}$ with the estimation technique a) Beamforming, b) MUSIC

Covariance matrix analysis showed similar features for both models (Eq. (9) and Eq. (15)) with the exception, that in near-field model the covariance matrix has Hermitian structure only and in the simplest - linear array model is neither periodical and has nor Toeplitz structure. Non-linear properties are defined by the non-linear functionality of the spacing of the sensors and sources (Eq. (14)). Nevertheless, the high level mathematical description apparatus remains the same. The decomposition of $\boldsymbol{W}$ into the steering-localization matrix $\boldsymbol{A}$ and the signal sources matrix $\boldsymbol{C}$ in the noncoherent situation for the far and near-field situation can be performed via the same known algorithms (i.e. Beamforming or MUSIC) [1, 2]. Defined parameters for the sampling frequency and the number of the samples gave satisfactory results. The results of the single source (Fig. 8) and two correlated sources (Fig. 9) in the near-field situations showed that the same localization estimation techniques can be used as in the far-field model. Unfortunately, in the near-field model for coherent-distributed signal sources situation "spatial smoothing" pre-processing, valid in the far-field model, is not applicable due to $\boldsymbol{W}$ non-linear properties. For such situation some different approach is needed.

\section{Acknowledgement}

This is extension work of project funded by the European Community's Seventh Framework Programme under Grant agreement 285202, "Safe control of noncooperative vehicles through electromagnetic means" (SAVELEC).

\section{References}

[1] GAŽOVOVÁ, S. and NEBUS, F. The Covariance Matrix Analysis for Near Field Sources Localization Model. In Proceedings of New Trends in Signal Processing. Demanovska Dolina: IEEE, 2018, p. 122-126. DOI 10.23919/NTSP.2018.8524098. 
[2] PILLAI, S.U. Array Signal Processing. New York: Springer, 1989. 221 p. ISBN 978-1-4612-3632-0.

[3] PISARENKO, F.V. The Retrieval of Harmonics from Covariance Functions. Geophysics Journal of the Royal Society, 1973, vol. 33, no. 3, p. 347-366. DOI 10.1111/j.1365-246X.1973.tb03424.x.

[4] GORBUNOVA, A.A. Stochastic Sources Localization Algorithm based on Near-Field Two-Point Planar Scanning Data (in Russian). Trudy MAI, 2014, no. 73. [viewed 201903-05]. Available from: http://trudymai.ru/eng/published.php?ID=48571

[5] MUDROŇ, J. Fundamentals of Classical Electrodynamics (in Slovak). Liptovský Mikuláš: Armed Forces Academy of General M. R. Štefánik, 2013. 263 p. ISBN 978-80-8040-480-2.

[6] NEBUS, F., KURTY, J. and MARKO, J. Controlled Spatial Smoothing for Coherent Signal Processing. In Proceedings of the Conference IST-039/RSY-011. Chester: ATO-RTO, 2003.

[7] CHEN, Z., GOKEDA, G. and YU, Y. Introduction to Direction-of-Arrival Estimation. Northwood, MA: Artech House, 2010. 193 p. ISBN 978-1-59693-089-6.

[8] WANG, H. and LIU, K.J.R. 2D Spatial Smoothing for Multipath Coherent Signal Separation Source. IEEE Transactions on Aerospace and Electronic Systems, 1998, vol. 34, no. 2, p. 391-405. DOI 10.1109/7.670322. 